PART I

SPHERICAL SYSTEMS 


\section{DYNAMICAL THEORY OF \\ SPHERICAL STELLAR SYSTEMS WITH LARGE $N$}

L. SPITZER, JR.

Princeton University Observatory, Princeton, N.J., U.S.A.

\section{The General Problem}

The dynamical evolution of a spherical star system is attractive to the theorist for two reasons. In the first place the physical problem, with some idealization, appears to have an appealing, if somewhat deceptive, simplicity; a large number of point masses, attracting each other with inverse-square gravitational forces, are assumed to move in a quasi-steady system with spherical symmetry. In the second place, the observed globular clusters appear to conform rather closely to this ideal of a spherical star system; to analyze the changes of these clusters with time, resulting from the dynamical interaction of stars with each other, is surely an important aspect of the theory of galactic evolution.

Some aspects of this evolution may be deduced from general statistical principles. Clearly the total energy of the system, including the kinetic energy, $T$, and the potential energy, $W$, must be constant. From the second law of thermodynamics we know that the direction of any dynamical evolution must be such as to increase the total entropy. In terms of statistical mechanics when energy is exchanged back and forth between particles in a steady state, with no energy input from the outside, the system will tend to evolve towards the most probable state, where the distribution of particles, or stars, among different energy states is in accordance with the probability $P_{i}$, computed from the equation

$$
P_{i}=C g_{i} \exp \left(-\lambda E_{i}\right),
$$

where $E_{i}$ is the energy of a star in some particular state and $g_{i}$ is the statistical weight of that state, proportional to the amount of phase space available; $C$ is a normalization constant, while the parameter $\lambda$ is inversely proportional to the mean energy of each particle, averaged over all particles.

The application of Equation (1) to a system of gravitationally interacting mass points runs into immediate difficulty because $P_{i}$ increases steadily as $E_{i}$ becomes more strongly negative, and there is no lower limit on $E_{i}$. Thus tightly bound gravitational subsystems may be expected to form and become even more strongly bound as a result of energy exchanges resulting from mutual encounters. Another difficulty is posed at the other end of the energy scale by stars for which $E_{i}$ is zero or slightly positive; the total phase space available for stars in such states may be regarded as infinite. Thus it is clear that a self-gravitating cluster has difficulty reaching a permanent equilibrium state. One final state appears to be possible, - two tightly bound 
stars revolving about each other in a permanent Keplerian ellipse, with all other stars escaping to infinity. This particular state is unchanging not because it has reached the maximum possible entropy, but rather because dynamical evolution has ceased, if we ignore gravitational radiation. In any case it is clear that in the evolution of a cluster a part of the system will expand, with perhaps some stars escaping, while another part will contract. If the simplicity of an isolated binary system is not reached, the contraction will continue until the initial assumptions are no longer valid. The finite stellar radius may be important if physical collisions occur, while general relativistic effects can be important if the escape velocity from the system becomes comparable with the velocity of light, as, for example, if material collapses toward a black hole.

We now list four different pathways by which a star system may evolve in conformity with Equation (1).

(1) Contraction of cluster as a whole, with escape or 'evaporation' of some stars.

(2) Contraction of inner core of cluster, with expansion at intermediate radii.

(3) Contraction of subsystem of heavier stars, with expansion for the lighter stars.

(4) Formation and contraction of binary systems.

A chief purpose of the dynamical theory summarized in the ensuing sections is to analyze the rate at which each of these processes occurs. In this introductory section we explain briefly the physical nature of each such process.

Process (1), first proposed by Ambartsumian (1938) and Spitzer (1940), is in many ways the simplest of the four, since it can be discussed quantitatively without any real evaluation of the internal structure of the cluster. Thus a moderately realistic estimate of the escape rate can be obtained with the entirely unrealistic assumption that the cluster is homogeneous and uniform. From the familiar virial theorem it is readily shown that the mean square value of the escape velocity, $v_{\infty}$, is given for an isolated system in general by

$$
\left\langle v_{\infty}^{2}\right\rangle=4\left\langle v^{2}\right\rangle
$$

Hence if a Maxwellian velocity distribution is established in the relaxation time, $t_{r}$, the fraction $\xi_{e}$ of stars that will gain enough energy to escape during this time is the fraction of the particles in a Maxwellian distribution, $P_{M}(v)$, which have velocities twice the rms value, $v_{m}$, giving

$$
\xi_{e}=\int_{2 v_{m}}^{\infty} P_{M}(v) \mathrm{d} v=7.4 \times 10^{-3} .
$$

As a result of this evaporation of stars, both the mass and energy of the cluster must decrease, with the cluster becoming progressively more tightly bound. For a homogeneous uniform cluster the evolutionary history can be traced (King, 1958b) until the system collapses entirely after a life of about 40 initial relaxation times. More elaborate dynamical investigations, using the Fokker-Planck equation for exact analytical computations of dynamical relaxation gave only slightly different results for homoge- 
neous clusters (Spitzer and Harm, 1958; King, 1965, 1966); for actual clusters the variations of density and relaxation time with position must also be considered (King, 1958a).

Process (2) can take at least two forms, depending on whether the energy released from the contraction of the core appears in the outer part of the cluster, or 'halo', or somewhere in the intermediate region. If the former, then an increasing number of stars will populate the halo with low binding energies and large apocentric distances. Such stars have relatively long periods and return only infrequently to the denser regions of the cluster. As we shall see in Section 3, the accumulation of stars in the outer halo is closely related to the escape of stars associated with process (1), and these two effects may conveniently be discussed together.

If the energy released by the core appears in the intermediate region of the cluster, process (2) takes a somewhat different form. To discuss this mechanism quantitatively requires rather detailed knowledge of the internal structure of the cluster. Analyses to date have been confined to rather idealized systems. In particular, Antonov (1962) and Lynden-Bell and Wood (1968) have considered this process in isothermal spheres confined within hypothetical rigid boundaries. The analysis assumed that the mean free path was short and thus, unlike the stellar case, the velocity distribution remained everywhere isotropic during the evolution. Their work demonstrated that such spheres would be thermally unstable if the central density exceeded the density at the boundary by a factor greater than 708 . In this situation, the system has the remarkable property that the entropy is increased by a development of a thermal gradient, with heat flowing from the contracting core to the expanding outer region. Since the kinetic energies of particles in a self-gravitating system increase as the system gives up energy and contracts, the contracting core becomes steadily hotter as it loses energy, while the outer region cools.

In actual clusters the physical situation is somewhat different from that assumed in these analyses - see Section 3 - but one might expect that perhaps a similar collapse of the central core - called the 'gravothermal catastrophe' by Lynden-Bell and Wood may occur if the density change within the isothermal region of the cluster amounts to more than some three orders of magnitude. There have been no computations of the rate to be expected for this type of collapse, but one might expect an accelerated collapse, since the relaxation time gets markedly shorter as the central core becomes denser.

Process (3), which Spitzer (1969) pointed out could lead to contraction without limit, results in a straightforward way from the tendency towards equipartition of energy between stars of different masses. During one relaxation time, $t_{r h}$, a heavier star should lose an appreciable fraction of its kinetic energy to the lighter stars and fall towards the center of the system, creating appreciable mass stratification. If there are only a few such heavy stars, they will reach equilibrium near the cluster center with lower velocities than the lighter stars and about the same kinetic energies. If the amount of mass in this concentration of heavy stars is appreciable, however, the selfattraction of these stars will require increased random velocities in equilibrium, 
equipartition with the lighter stars becomes impossible, and the subgroup of heavy stars will continue to lose energy to the lighter stars, continually contracting with increasing velocities and increasing departure from equipartition. The initial time scale for this type of collapse should be of the order of the relaxation time in the inner regions of the cluster, but in contrast to the evaporation and gravothermal catastrophes there is no reason to expect any acceleration of the collapse. Indeed, if kinetic energy is lost by the heavy stars at a constant rate, which would be expected if the density and rms velocity of the light stars remained constant, the mean value of $M / r$ for these stars might be expected to increase at a constant rate, giving $\mathrm{d} r_{1} / \mathrm{d} t$ varying as $-r_{1}^{2}$, if $r_{1}$ is the mean radius for this subgroup.

Process (4) was pointed out by Aarseth (1968) and van Albada (1968) as a major influence on the evolution of systems with some 25 to 250 stars. Numerical integration of the exact equations of motion for such systems, following von Hoerner $(1960,1963)$, showed that binary stars were formed and disrupted, but that one or more tightly bound binaries generally accounted for most of the binding energy of the cluster at the end of the evolutionary period followed. General arguments - Spitzer and Hart (1971a), referred to as Paper I - show that the three-body encounters which form tightly bound binary systems become less and less important as the number of stars, $N$, increases. A detailed analysis by Heggie (1974) verifies this result and shows that in a cluster of $10^{5}$ stars, for example, the formation of binaries should be quite unimportant for dynamical evolution. We shall therefore neglect process (4) throughout most of the discussion below.

As emphasized by Heggie (1974), there are two situations in which absorption of energy by binary systems can be important even in clusters with large $N$. Firstly, if the core of the cluster collapses, the formation of binaries may become important when a relatively small number of stars is involved in a high-density core; we return to this subject again in Section 6. Secondly, if a sufficient fraction of the stars are binary from the beginning, with a separation of a few astronomical units between components, the dynamical evolution may be profoundly affected, with the cluster as a whole perhaps even expanding rather than contracting. It has often been assumed that binaries are relatively infrequent in systems of population type II, and indeed very few binaries have been detected in globular clusters. However in van de Kamp's (1971) list of stars within $5.2 \mathrm{pc}$, the fraction which are in fact multiple systems is about $40 \%$ independent of space velocity. Thus of the 13 stars with a heliocentric velocity known to exceed $70 \mathrm{~km} \mathrm{~s}^{-1}, 3$ are double and 2 are triple systems. Of the remaining 32 stars, with lower (or uncertain) velocities, 13 are double stars, with 1 triple. The possible presence of such multiple systems in globular clusters has so far been generally ignored and must certainly be considered in future work.

\section{Dynamical Methods for Large- $\boldsymbol{N}$ Systems}

Since it is not feasible to integrate numerically the exact equations of motion for a system with a very large number of stars, an approximate solution must be sought. As 
the basis for such an approximation in spherically symmetric systems we may express the gravitational acceleration $\mathbf{g}_{i}$, for any star $i$, as the sum of two terms;

$$
\mathbf{g}_{\mathrm{i}}=\frac{\mathrm{d} \mathbf{v}_{i}}{\mathrm{~d} t}=-\mathbf{r}_{1} \frac{\partial \varphi\left(r_{i}, t\right)}{\partial r}+\mathbf{F}_{i}(t),
$$

where $\mathbf{r}_{1}$ is a unit vector in the $r$ direction. The radial potential function, $\varphi(r, t)$, may be computed from the smoothed distribution of stars; if the system is in a quasi-steady state, $\varphi(r, t)$ will change only slowly with time. The function $\mathbf{F}_{i}(t)$ represents for each star the difference between the actual acceleration and the smoothed spherically symmetric value, and results from the granularity of the actual distribution of mass points. The basic approximation made is that the effects produced by this granularity may be computed from an analysis of successive two-body encounters. This approximation is believed to be relatively accurate, although for some effects, such as the formation of binaries, one must consider encounters of three bodies and other higherorder correlations.

The analytical theory of two-body encounters has been thoroughly explored by Chandrasekhar (1942), and by Rosenbluth et al. (1957). However, to apply this theory to the complex configuration of a stellar cluster seems discouragingly complicated, see Hénon (1973). Instead, Monte Carlo techniques have been used to follow numerically the orbits of some thousand 'test stars', taken to be a representative sample of a larger group. In these techniques the orbit of each star in the potential field, $\varphi(r, t)$, is taken into account, and velocity perturbations are applied with suitable random sampling techniques so that the net effect of two-body encounters is correctly given. Two different methods have been published, the first by Henon $(1967,1971,1973)$, the second by a group at Princeton (see Spitzer and associates, Papers I-VII). These methods differ both in the consideration of stellar orbits in the potential field $\varphi(r, t)$ and in the choice of velocity perturbations resulting from $\mathbf{E}_{i}(t)$.

In the work by Hénon the unperturbed stellar orbits are not actually computed in detail. For his Monte Carlo computations the only information required from the orbits is the resultant probability distribution of $r_{i}$, the star's distance from the center, and this can be determined from the star's energy, $E$, and angular momentum $J$ (both taken per unit mass) if the potential field $\varphi(r, t)$ is known. For each of the test stars a particular position in its orbit is determined, in accordance with this probability distribution. Adjacent stars are then assumed to have a mutual encounter, changing the velocity of each. All the geometrical angles in this encounter are chosen at random, but the collision parameter, $p$, (the distance of closest approach in the absence of mutual forces) is computed to give the correct total mean square deflection, considering the local density, the time step used and the actual relative velocity of the two stars. After each such collision, new random positions are chosen for each of the two stars, taking into account the new $E, J$ of each and the potential $\varphi(r, t)$ is recomputed; spherical symmetry is assured by the assumption that each star considered is one of a large number of stars all with identical radial and transverse velocities and all arranged in a spherical shell (called a 'superstar' by Hénon). 
In the computations at Princeton, (see Spitzer and associates, esp. Papers I, IV and VII) Equation (4) is integrated numerically with time to give $r_{i}(t)$ for each of the 1000 test stars; again, for the computation of $\varphi(r, t)$ each test star is assumed to represent a large number of similar stars arranged in a uniform spherical shell. The velocity perturbations are computed in groups rather than from individual two-body collisions as in Hénon's method. The cluster is divided into 25 spherical shells or regions, each one containing 40 test stars; the mean density $\varrho$ and rms velocity $v_{m}$ are determined in each region, with separate values for stars of each mass group. For each star of velocity $\mathbf{v}$, the velocity perturbations are assigned according to a Gaussian distribution formula which gives the correct first and second moments of $\Delta \mathbf{v}$ for a star of that mass and velocity interacting separately with the stars of each mass group; each such group is assumed to have a Maxwellian distribution of velocities, with the rms velocities and densities computed for that group of field stars in the region considered.

In the Princeton computations the ratio of the relaxation time, $t_{r h}$, to the mean crossing time, $r_{h} / v_{m}$, is taken to be in the range from 10 to 50 , corresponding to a cluster with $N$ between 500 and 4000 . For clusters with so small a value of $N$ the use of a smoothed radial potential becomes questionable, and the Monte Carlo computations are not completely valid. For clusters with $N$ equal to $10^{5}$, the actual ratio of $t_{r h}$ to $r_{h} / v_{m}$ (which varies as $N / \log (0.4 N)$ - see Paper I) is about 800 , and the computing time involved in following stars for so many orbits would be prohibitively large. Fortunately, most aspects of cluster evolution, as measured in units of $t_{r h}$, are independent of the crossing time, a fact which forms the basis of Hénon's method, and hence the Princeton computations can be taken as representing the dynamical history of clusters with large $N$. In considering the escape of stars from the cluster, an effect whose detailed mechanism depends on the ratio of relaxation to crossing time and hence on $N$, a separate theoretical study indicates the modifications needed in the computed models (see Section 3.3.).

Comparing these two methods, the one developed by Henon has the advantage that it requires somewhat less computing time. In addition, the computation of velocity perturbations takes the exact distribution of stellar velocities into account; this latter advantage is not too significant, since in the denser regions where encounters are significant the actual distribution is generally rather closely Maxwellian. The Princeton method has some advantage in that individual orbits are followed. Initial dynamical collapse can be considered and, as pointed out above, a basis is given for analyzing the effect of distant encounters in producing escape of stars, which cannot be considered directly with Hénon's method.

\section{Results for Isolated Systems with Stars of Same Mass}

\subsection{REFERENCE RELAXATION TIME}

In this section we shall give some results for the simplest possible system, obtained with Monte Carlo calculations, mostly by the Princeton group. It is often convenient to express time in units of a reference relaxation time, defined as the relaxation time 
at the mean density for the inner half of the cluster mass, $M$, with stars assumed to have the rms velocity of the cluster as a whole. With some simplifications and approximations, - discussed in Paper I - we obtain

$$
t_{r h}=\frac{0.060 M^{1 / 2} r_{h}^{3 / 2}}{m G^{1 / 2} \log (0.4 N)}
$$

where $r_{h}$ is the radius containing half the mass, $N$ is the total number of stars, and $m$ is the mean mass, equal to $M / N$. The median radius, $r_{h}$, changes relatively little during the evolution of most of the models; in any case it is simplest to use the initial value of $t_{r h}$ as a reference parameter throughout the life of the cluster. Because of the tremendous central concentration of most clusters, the local relaxation time can be several orders of magnitude shorter than $t_{r h}$ at the center and enormously greater in the far halo.

\subsection{DEVELOPMENT OF ISOTHERMAL SPHERE AND SURROUNDING HALO}

As noted above, one would expect collisions to establish a velocity distribution close to the Maxwellian in the central regions of the cluster. The Monte Carlo models may be used to show the actual value of $f(E)$, the density of test stars in phase space, as a function of $E$, the star's energy per unit mass. Figure 1, taken from Paper IV, shows the values obtained in the inner three regions (containing the inner $12 \%$ of the mass) in one of the model one-component systems. The energy scale is defined so that a star at rest at infinity has zero energy. In the outer regions, the phase density should be a function of $J$ as well as of $E$, but in the central regions the velocity distribution is isotropic and $f$ is a function of $E$ only.

The points mostly lie close to the solid line, representing an exponential decrease of $f(E)$ with increasing $E$. However, this distribution would give a finite value for $f(0)$, giving much too large an escape rate. For $E$ only slightly negative, the points lie reasonably close to the dashed line, representing the 'lowered Maxwellian'

$$
f(E)=C \exp \left[\left(-3 E / v_{m}^{2}\right)-1\right] \text {, }
$$

proposed by Michie (1963) and King (1965); $C$ is a constant of proportionality. Equation (6) is based on the assumption that the energy required for escape is zero. This lowered Maxwellian distribution seems a reasonably good approximation for the cluster models. At values of $E$ between the last plotted point and zero, the phase space density drops to a value much below that given in Equation (6). The statistical weight of these states of slight negative energy is relatively very large, and the gradual increase of $f(E, 0)$ in these states as time goes on is an important part of the cluster evolution, which leads, as we shall see below, both to the development of the halo and to escape of some stars.

As a result of this tendency towards an isotropic Maxwellian distribution, the inner half of the cluster, within the radius $r_{h}$, approximates the inner region of an isothermal sphere. There are slight deviations from strictly isothermal conditions because of the drop in the velocity distribution function at energies near zero, discussed above. 
However, in the central regions this drop is way out in the wings of the velocity distribution function and produces little direct effect locally, except for a slight decrease of the rms velocity with increasing radius.

In the halo a quite different situation prevails. Since the local dynamical relaxation time is very long, the velocity distribution is anisotropic. The phase space density is

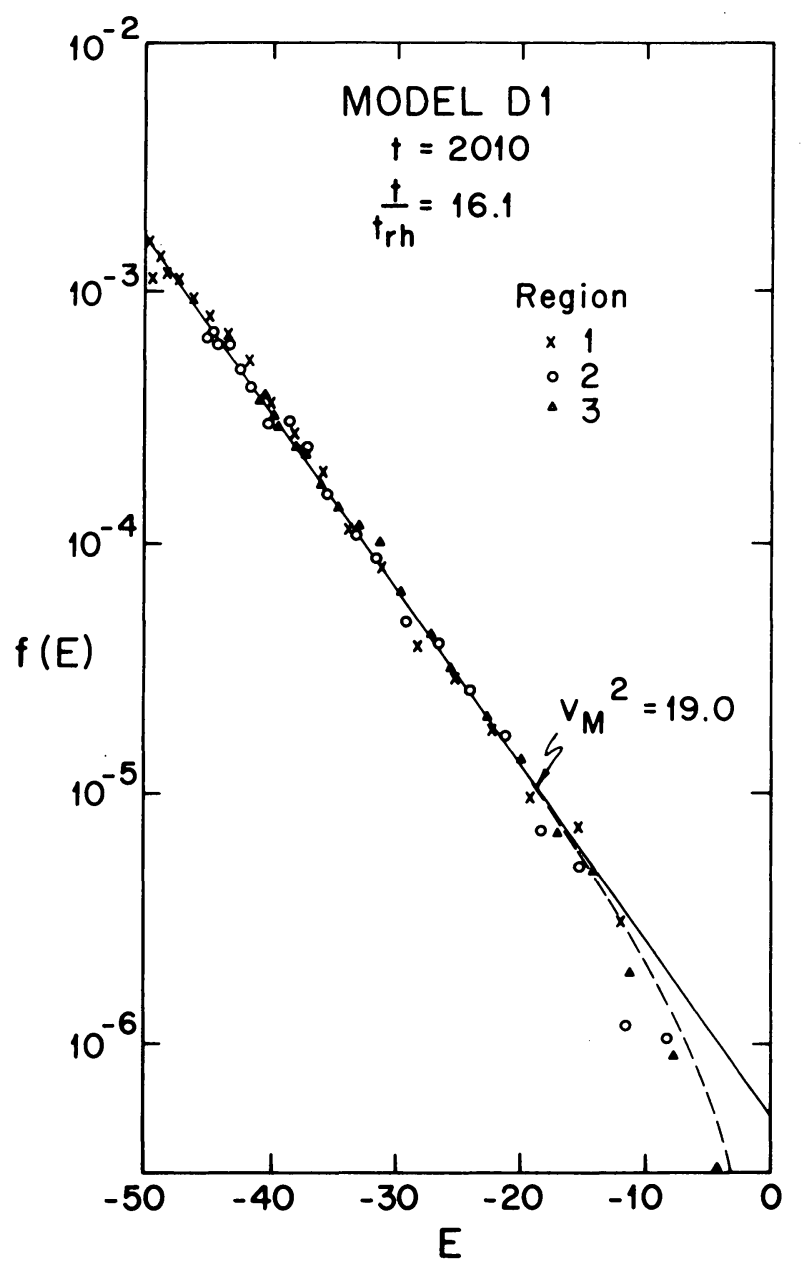

Fig. 1. Density in phase space, $f(E)$, as a function of stellar energy, $E$ (potential plus kinetic), in the inner regions of Model D1 fairly late in its development. The straight line represents the usual Maxwellian distribution, while the dashed line represents the lowered Maxwellian distribution (Equation (6) in text), which decreases linearly to 0 as the energy approaches 0 , the value for a star motionless at infinity.

constant along a dynamical trajectory when collisions are infrequent, and hence along orbits which intersect the core, $f(E, J)$ has the same values as in the core. For $J$ greater than a certain critical value, the orbits are restricted to regions of low density, outside the isothermal core, and if these orbits are not populated originally, they will remain unpopulated. Thus the halo contains stars on predominantly radial orbits, 
with transverse velocities varying as $1 / r$ since the angular momenta remain constant with increasing distance.

To obtain the density in the halo we integrate $f(E, J)$ over $2 \pi v_{t} \mathrm{~d} v_{t} \mathrm{~d} v_{r}$, where $v_{t}$ and $v_{r}$ are the tangential and radial velocities, respectively. As a reasonable first approximation we may set $f(E, J)$ equal to $f(E, 0)$ for $J$ less than some upper limit, $J_{1}$, and zero outside. Thus $f(E, J)$ differs from zero only within a range * $\Delta v_{t}$ from 0 to $J_{1} / r$, and we have

$$
\varrho(r)=\pi\left(\Delta v_{t}\right)^{2}\left(\Delta v_{r}\right) f,
$$

where $f$ is a mean value of $f(E, 0)$ over $v_{r}$, taken over the range ${ }^{*} \Delta v_{r}$ from 0 to the escape velocity $(2 G M / r)^{1 / 2}$. According to Equation (6), expanding the exponential for

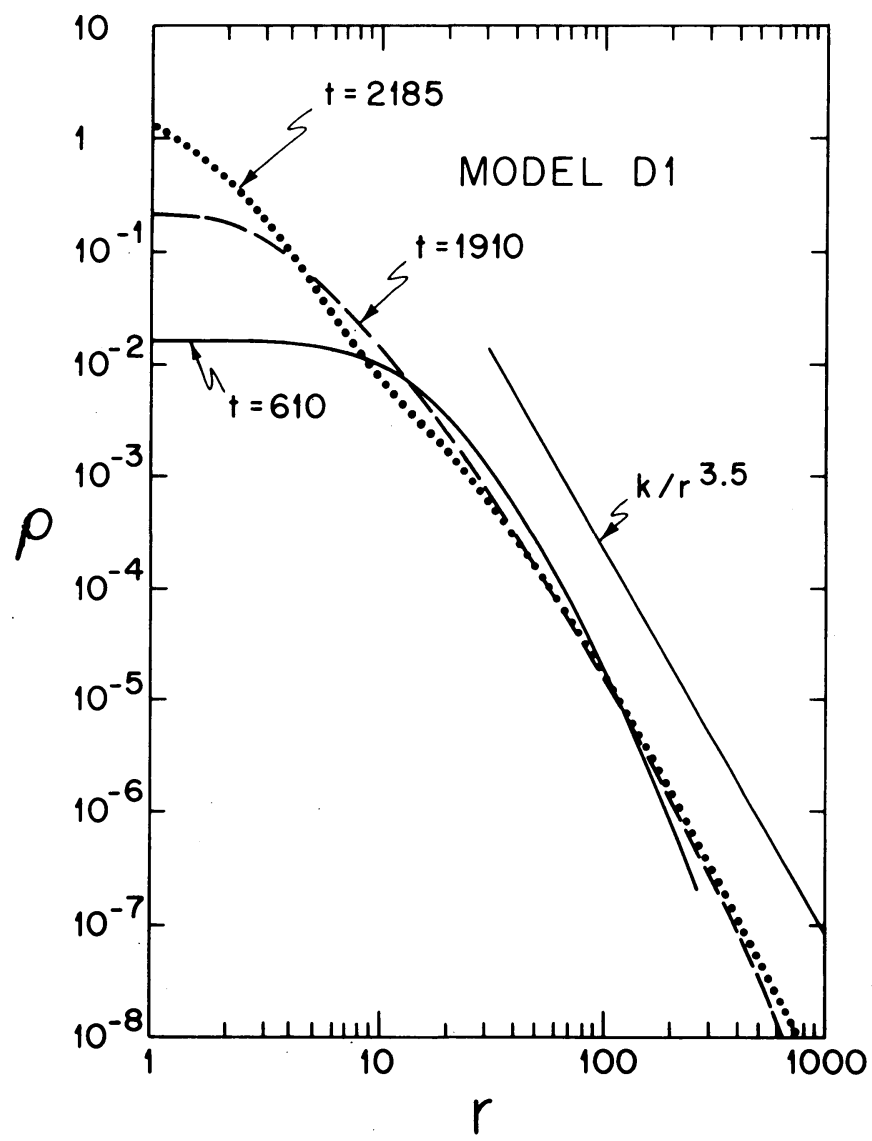

Fig. 2. Density distribution, $\varrho(r)$, as a function of distance $r$ from the center at different times in the development of Model D1. The straight line at the right represents the theoretical density distribution in a well developed halo. This model starts with an initial collapse, which generates a substantial halo at the beginning. The dimensionless units for $\varrho$ and $r$ are explained in Paper II.

* The velocity ranges $\Delta v_{t}$ and $\Delta v_{r}$ in Equation (7) should not be confused with the velocity perturbations which are denoted by these same symbols in Paper IV. 
small $E, f$ decreases from $-3 C E / v_{m}^{2}$ to zero as $v_{r}$ increases over this range; since $-E$ equals $G M / r$ when $v_{r}$ vanishes, $f$ is proportional to $1 / r$. Since, as we have seen, $\Delta v_{t}$ and $\Delta v_{r}$ vary as $1 / r$ and $1 / r^{1 / 2}$, respectively, Equation (7) yields

$$
\varrho(r)=k / r^{3.5} \text {, }
$$

where $k$ is a constant of proportionality.

The density distributions in two different models at successive times are plotted in Figures 2 and 3, which show the isothermal central region and the outlying halo. The

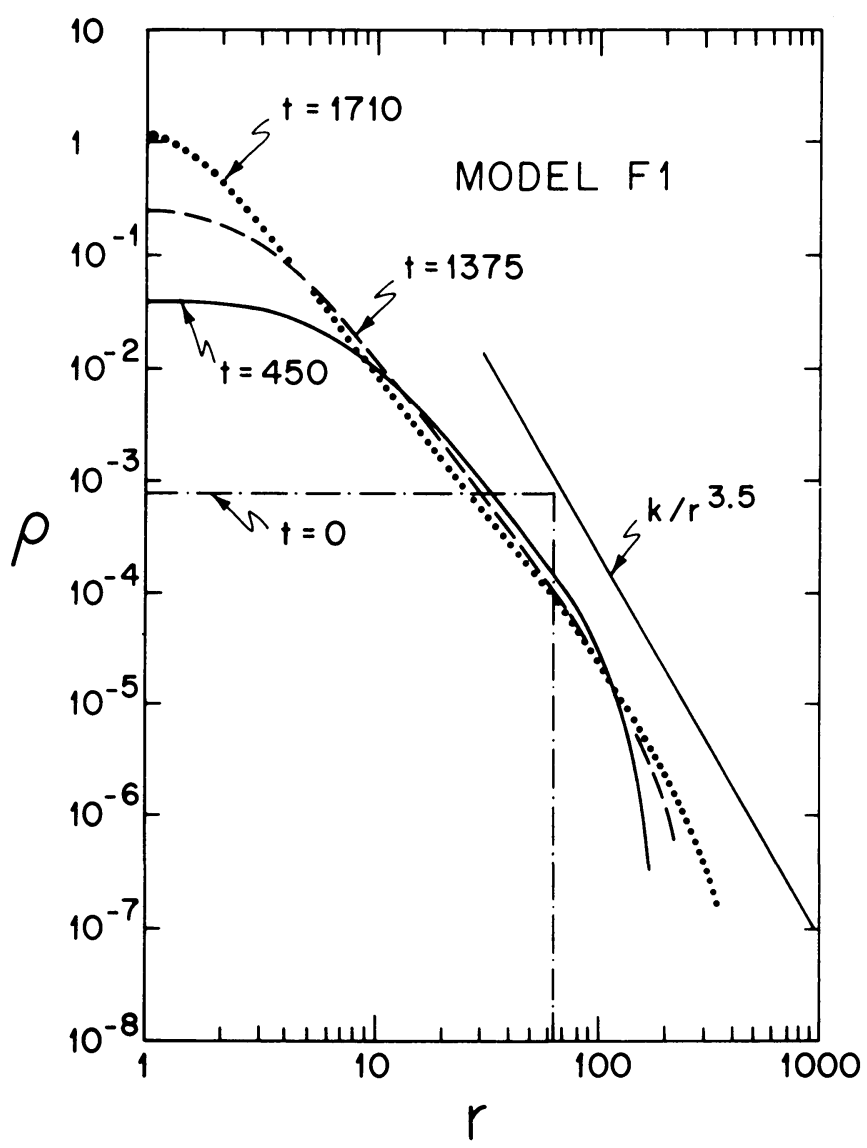

Fig. 3. Density distribution $\varrho(r)$ at different times in the development of Model F1. This model starts as a sphere of constant density in equilibrium, with all stars in circular orbits. There is no appreciable halo at the beginning, and the halo develops much later than in Model D1, shown in Figure 1.

upper right solid lines represent Equation (8), which seems to provide a good fit for the halo. The progressive contraction of the isothermal core, which is discussed in more detail below, is evident in these figures. The outward growth of the halo is also evident. For stars in the halo the mean value of $J$, the angular momentum of each star, does not change appreciably with time, nor does the value of the constant $k$ in Equation (8). 
Halo growth consists of extending the range of $r$ over which Equation (8) is valid; at greater $r$ the density drops off very sharply. In terms of $f(E, 0)$ the development consists of increasing the phase-space density at energies only slightly negative up to the value given in Equation (6). Halo growth is particularly evident for Model $F$, for which the initial equilibrium configuration is a sphere of constant density, with no halo whatever, and therefore the entire halo must develop as a result of two-body encounters. Model D starts off with an initial collapse, which produces a substantial halo to begin with, and the outward growth of the halo is less noticeable.

One way of presenting the Monte Carlo data on evolution of a cluster is to plot the radii containing different fractions of the total mass as functions of the time. Such a plot for Model F is given in Figure 4, where the solid line gives the results obtained by Hénon, while the individual points show Princeton results from Paper IV. The close agreement is very gratifying. The outward extension of the halo and the contraction of the core are both evident.

\subsection{ESCAPE OF STARS}

Stars can escape from a cluster by a close encounter with another star at any position within the cluster. Such encounters produce velocity changes comparable with the relative velocity of the two stars, and in view of Equation (2), velocities exceeding $v_{\infty}$ can result. The resultant rate of escape for the cluster as a whole has been computed by Hénon (1969).

In clusters with large $N$ the cumulative effect of many distant encounters, producing small deflections, is more important in leading to escape of stars. In any one orbit the change of energy is small, leading in the first instance to outward diffusion of the stars and growth of the halo rather than to escape. We analyze the conditions under which halo stars can actually escape. We may define $\varepsilon_{2}$ as the root mean square change of energy of a halo star during one orbit from apocenter, where $r=r_{a}$, through the isothermal core and back again to apocenter. Evidently $\varepsilon_{2}$ will increase as $J$ decreases, increasing the density experienced by the star at pericenter. For each $J, \varepsilon_{2}$ will not depend significantly on $E$ for halo stars, when $E$ is only slightly less than zero. There will also be a mean change of energy per orbit, denoted by $\varepsilon_{1}$, but this is smaller and we may neglect it. Evidently when the energy of the halo star, closely equal to $-G M / r_{a}$, equals $-\varepsilon_{2}$, escape is somewhat likely. We define this value of $r_{a}$ as the critical distance, $r_{f}$. As $r_{a}$ increases above $r_{f}$, the probability of escape per orbit approaches 0.5 .

Let us now consider what happens when $N$, the total number of stars, increases. We keep the total mass $M$ and the cluster dimensions constant, varying only the mean mass per star: thus the stellar energy per unit mass and the potential function $\varphi(r)$ remain unaffected. In this case it may be shown - see Paper II - that $\varepsilon_{2}$ decreases as $1 / N^{1 / 2}$, while $\varepsilon_{1}$ varies as $1 / N$. Hence the critical radius $r_{f}$ increases as $N^{1 / 2}$, and the halo development must proceed to greater radial distances before escape becomes possible. This development to large $r$ is limited by the long periods of the extended halo orbits. The period of a star with apocenter distance $r_{a}$ is proportional to $r_{a}^{-3 / 2}$, and thus $P_{f}$, the period at the critical distance, increases as $N^{3 / 4}$. Since the time 
required for evolution by encounters varies as $N$ (the small variation of $\log N$ is ignored here), diffusion of halo stars out to the critical distance occurs more readily as $N$ is increased, even though the critical distance increases with $N$. It may be shown that after the halo has grown out to the critical radius, the rate of escape per unit $t_{r h}$

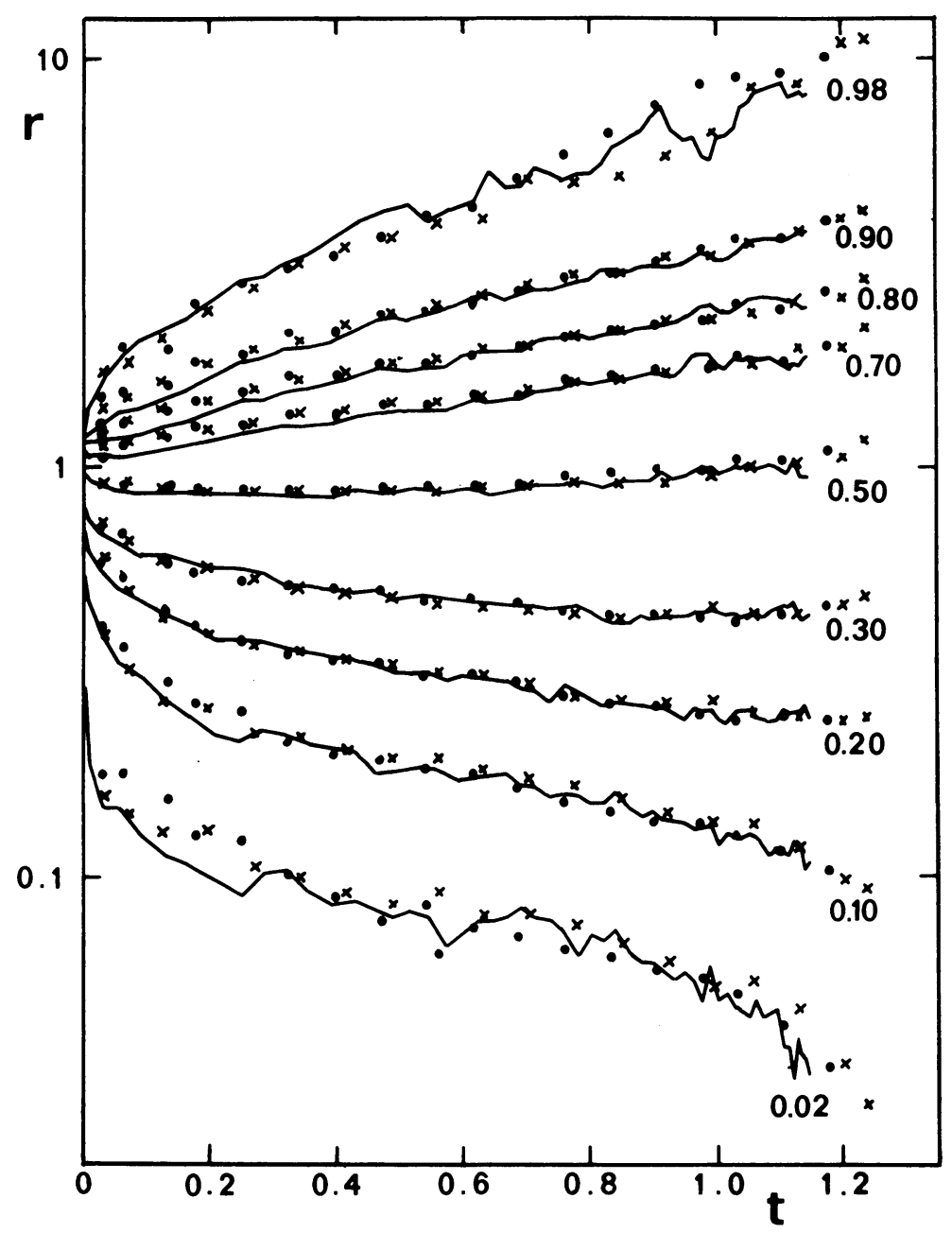

Fig. 4. Values of the radius containing various fractions of the total mass of Model F, plotted against the dimensionless time used by Hénon. The solid line represents the computations by Hénon (1973) for this model, while the dots and crosses represent values obtained by Spitzer and Thuan (1972) for models F1 and F2, respectively; these two models differ only in the ratio of the relaxation time, $t_{r h}$, to the dynamical crossing time, $r_{h} / v_{m}$, which equals 10 and 20 , respectively, for $F 1$ and F2.

in independent of $N$, although the mean energy of the escaping stars, which equals $0.58 \varepsilon_{2}$ - see Paper III - will decrease as $1 / N^{1 / 2}$.

The Monte Carlo calculations at Princeton give quantitative results in accord with these theoretical expectations. Thus in Model F, where there is no halo to start with 
and the halo does not become fully developed until near the end of the computations, no stars escape during the first $60 \%$ of the period covered, and during the last $10 \%$ of the time the escape rate reaches about $0.3 \%$ per time interval $t_{r h}$. In Model D, where the halo develops much earlier, the escape rate reaches this same value about $55 \%$ of the way through the evolutionary time followed. The value of $\varepsilon_{2}$ in Model D1 is about 2 for $J=100$, a representative mean value for the halo, giving a highly eccentric orbit. Thus $r_{f}$ is about 500 for this model, with about the same value for Model F1 also. The corresponding period, $P_{f}$, is 800 time units (for $G=1$ and $M=10^{3}$, in the dimensionless units used). The halo in Model D1 becomes well developed out to this radius well before the end of the computations, while in Model F1, this stage is reached only very near the end. Separate computations in which $N$ was varied, keeping all other quantities the same, confirm that the mean energy of escaping stars varies about as $N^{-1 / 2}$, and is roughly equal to $\frac{1}{2} \varepsilon_{2}$.

The measured rate of escape, $\xi_{e}$, of about $3 \times 10^{-3}$ per interval of $t_{r h}$ is less than computed from Equation (3) by a factor 0.4 . If somewhat more sophisticated methods are used for predicting $\xi_{\boldsymbol{e}}$, the reduction factor is slightly smaller, about 0.3 . This discrepancy is largely accounted for by the rate at which stars accumulate in the outermost halo; in Model D1 this rate is about twice the measured $\xi_{e}$. These stars return to the isothermal core so infrequently that they are very similar to escaping stars and should really be grouped with them for some purposes. For systems with $N$ as large as $10^{5}$, the halo would be more fully developed, the ratio of stars accumulating in the far halo to those escaping, per unit time interval, would fall below unity late in the evolutionary development, and $\xi_{e}$ would be closer to the predicted value of about 0.01 .

In terms of these results it is clear why Hénon's method does not give the rate of escape. The dynamical time in which a star moves around its orbit from apocenter to apocenter does not appear in his method, which corresponds to the limiting case of infinite $N$. Thus the rms change of energy in a single orbit is not considered, and there is no way of determining the value of $r_{f}$ beyond which stars will begin to escape. His technique gives correctly the total rate at which stars diffuse up towards zero energy, but cannot distinguish evaporation at slight positive energy from halo buildup at slight negative energy. The Fokker-Planck diffusion equation, based on the limit of infinitesimal velocity changes, gives no escape of stars from an isolated cluster. As shown in detail in Paper III, consideration of finite velocity changes in the neighborhood of zero $E$ is required to give correctly the escape rate which is, in fact, present.

One may ask what the present theory should predict for the process of escape from systems with smaller $N$. If $N \approx 250, \varepsilon_{2}$ is about $60 \%$ of the mean kinetic energy per unit mass of the cluster stars. If the present theory is still applicable for systems of such low $N$, which it may conceivably be at times before the presence of binaries dominates the exchange of kinetic energy between stars, the mean energy of the escaping stars (their kinetic energy at infinity) should be about a third of the mean kinetic energy of the cluster stars, in rough agreement with the results obtained by Aarseth (1975) for one-component systems with $N=250$. Detailed numerical agreement is not to be 
expected, since the standard theory for large $N$ ignores terms whose relative order is $1 / \ln N$. However it is evident that for small $N$ the exchange of energy in one orbit is relatively large, in contrast with the conventional velocity diffusion picture which must be applicable for large $N$.

\subsection{CONTRACTION OF ISOTHERMAL CORE}

In all the Monte Carlo models the core contracts steadily throughout the evolution. At the beginning, the driving force behind this contraction for the one-component models is apparently the diffusion of stars toward zero energy in response to the drop of $f(E)$ below the Maxwellian value for slightly negative energies (shown in Figure 1). A detailed energy accounting in Paper IV shows that during most of the cluster's evolution the energy gained by stars which accumulate in the halo at slight negative energies or which escape at slight positive energies is just about balanced by the energy given up by the contraction of the inner core. Furthermore, the rate of contraction is about that predicted from the simple evaporation theories. As we have seen, these neglect halo formation, but they give correctly the rate at which stars accumulate at energies near zero; whether a star ends up orbiting in the halo or escaping from the system does not affect much the energy it has required to raise it above the average stars.

Towards the end of the evolutionary calculation the contraction seems to change its character. The rate of contraction, as measured at the radius containing $2 \%$ of the mass, actually accelerates, although the rate at which stars escape and accumulate in the halo does not seem to change very much. In Figure 5 the radii containing $2 \%$ and $10 \%$ of the mass of Model $\mathrm{F}$ are plotted against time. Evidently the squares of these radii, proportional to the spherical areas at these radii, decrease linearly with time, and seem destined to reach zero in a finite time. The time interval between the start of the cluster's evolutionary history and this final collapse of the central region is denoted by $t_{\text {coll }}$, and is from 12 to 19 times $t_{r h}$ in different isolated clusters. Somewhat similar results were obtained by Larson (1970) with an approximate analytical theory.

A detailed energy accounting for Model D (see Paper IV) confirms that the rate of energy release from the core very late in the evolution is greater than can be accounted for by diffusion of particles up to small positive or negative energies. Instead the energy released near the center goes into readjustment of intermediate regions of the cluster. In view of the relatively small numbers involved, this result is not very secure statistically. Just what is happening within the cluster is not clear in detail, but one is tempted to identify this final collapse with the gravothermal instability described as process (2) in Section 1. On this basis one would expect the range of density variation within the isothermal sphere to surpass some critical value before the final collapse. Since there is a gradual transition between the isothermal central regions and the halo, this expectation cannot be tested precisely. However, in the later stages of development the median radius, $r_{h}$, provides a rough approximation for the radius of the isothermal zone. The ratio of the central density, $\varrho(0)$, to $\varrho\left(r_{h}\right)$, the density for $r$ equal $r_{h}$, attains the value $10^{3}$ relatively late in the collapse of the one-component models analyzed, 
and a gravothermal catastrophe under these conditions would seem a logical inference.

On the other hand, it must be admitted that the physical situation in a cluster of large $N$ is quite different from that envisaged in the theoretical analysis of bounded isothermal spheres of gas. In addition to the lack of a rigid boundary, the local dynami-

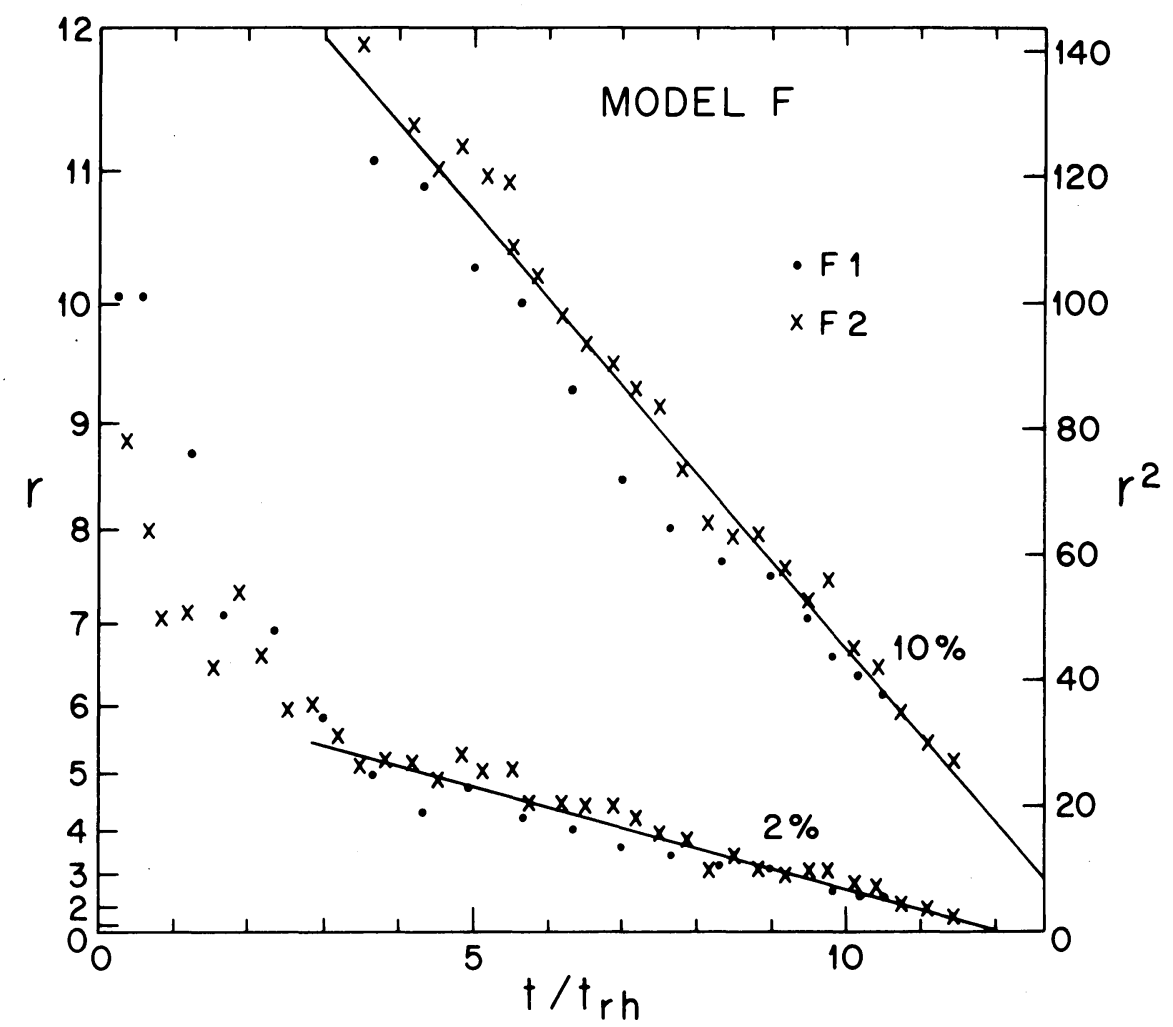

Fig. 5. Values of the radius containing $2 \%$ and $10 \%$ of the total mass of Model F, replotted from Figure 4. As before, dots and crosses represent the results for Models F1 and F2, respectively. The straight lines represent empirical fits to the data.

cal relaxation time is much longer than the dynamical crossing time, or the comparable orbital period. Thus the phase space density will be constant along each dynamical trajectory. Any departure from thermal equilibrium in the intermediate region of the cluster will therefore appear not in a perturbation of a local temperature, with radial temperature gradients appearing, but rather in a difference of $f(E, J)$ between orbits of the same $E$ but different $J$; i.e., in a developing anisotropy of the velocity distribution. More generally, Miller (1973) has argued that the thermodynamic considerations which lead to the gravothermal instability are not directly applicable to stellar systems. More analysis is clearly required, and the present tentative identification of the gravothermal instability is suggestive rather than conclusive. 
If this identification were correct, one would naturally question what role the gravothermal instability should play in systems of relatively small $N$, which have been followed by direct integration of the exact equations. There is some question whether this effect should appear when $N$ is between 100 and 250. In the Monte Carlo models, this instability appears when the density within the inner $2 \%$ of the cluster mass is some 1000 times as great as the lowest density in the isothermal region. Such a mean density becomes difficult to define when the number of stars involved is as low as 2 , and even with as many as 5 stars, there is some question as to how good the correspondence should be between an actual system and a model based on an essentially continuous distribution of matter.

Quite apart from the detailed mechanisms involved, one may ask why the area of the spheres containing a small fixed percentage of the total mass should decrease linearly with time during the final collapse, as indicated in Figure 5. It was shown in Paper IV that this result follows approximately if one assumes that the contracting core loses a certain fraction, $\eta$, of its kinetic energy during each relaxation time at the center. The energy lost is presumably conducted away to more distant regions of the cluster; the core contracts, of course, as a result of the energy drain, and the gravitational energy so released heats the core to higher rms velocities than before. Numerical values in Paper IV for the collapse rates indicate that on the average

$$
\langle\eta\rangle=2.2 \times 10^{-3},
$$

with an average deviation of about $10 \%$ for the three models of isolated systems.

\section{Results for Systems with a Distribution of Stellar Mass}

\subsection{Development of Mass Stratification}

If two groups of stars are present, each with a Maxwellian velocity distribution with rms velocities $v_{m 1}$ and $v_{m 2}$, and with masses $m_{1}$ and $m_{2}$, then encounters between the two groups of stars in a region where the particle densities are $n_{1}$ and $n_{2}$ will change the mean energy, $E_{m 1}$ of group 1 stars at a rate (Spitzer, 1969)

$$
\frac{\mathrm{d} E_{m 1}}{\mathrm{~d} t}=\left(\frac{1}{2} m_{2} v_{m 2}^{2}-\frac{1}{2} m_{1} v_{m 1}^{2}\right) / t_{\mathrm{eq}}(1,2),
$$

where

$$
t_{\mathrm{eq}}(1,2)=\frac{\left(v_{m 1}^{2}+v_{m 2}^{2}\right)^{3 / 2}}{8(6 \pi)^{1 / 2} n_{2} m_{1} m_{2} G^{2} \ln (0.4 N)} .
$$

Conservation of energy requires that

$$
\frac{t_{\mathrm{eq}}(1,2)}{n_{1}}=\frac{t_{\mathrm{eq}}(2,1)}{n_{2}}
$$

The ratio of the 'equipartition time' $t_{\mathrm{eq}}$ to the local relaxation time $t_{r}(2,2)$, for en- 
counters among stars of group 2, is given by

$$
\frac{t_{\mathrm{eq}}(1,2)}{t_{r}(2,2)}=0.44 \frac{m_{2}}{m_{1}}\left(1+\frac{v_{m 1}^{2}}{v_{m 2}^{2}}\right)^{3 / 2} \text {. }
$$

If the lighter stars, of mass $m_{2}$, predominate, the relaxation time $t_{r}$ for the entire system is close to $t_{r}(2,2)$, and the equipartition time for the heavies will be somewhat shorter than $t_{\boldsymbol{r}}$.

One would expect that in a system with an initial distribution of masses the tendency towards equipartition would produce a marked stratification within a time comparable to $t_{r h}$. Early Monte Carlo computations reported in Paper II demonstrated this effect. Several rather serious approximations made in this work have now been improved, - see Paper VII. Figure 6 shows the mass fractionation in a cluster

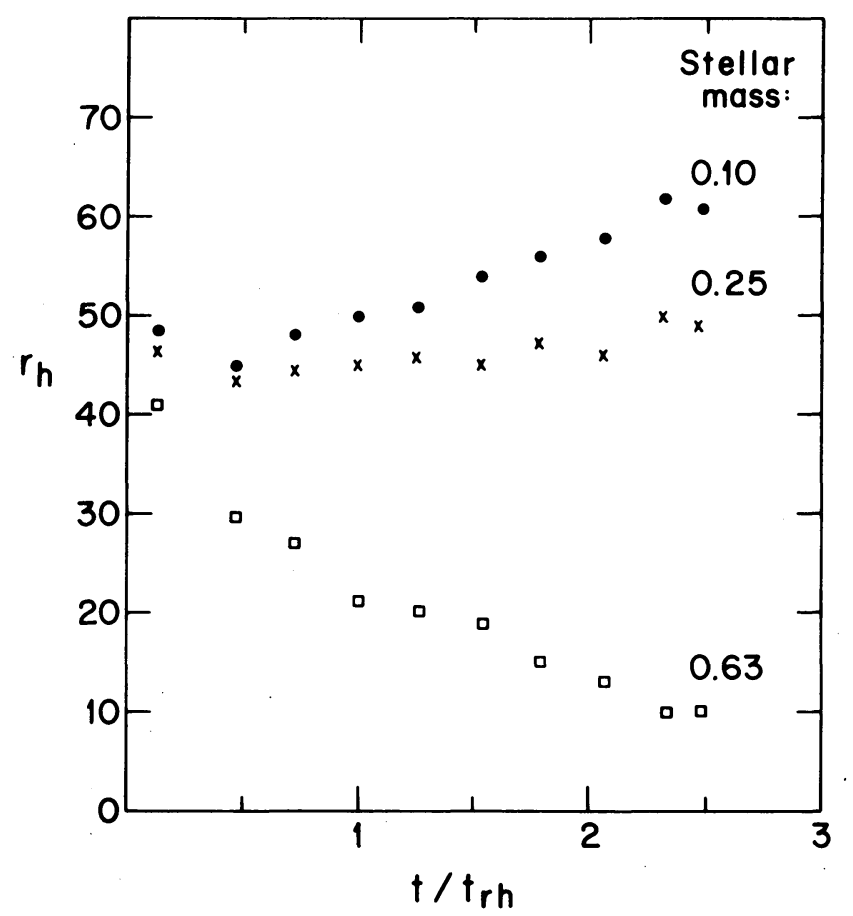

Fig. 6. Radius containing half the mass for each of three mass groups in Model La, plotted as a function of time in units of $t_{r h}$. The stellar masses given are ten times the values used in the computer program and, in solar masses, give representative values for old systems of population type II.

with three components, (Model La) with the mass distribution spectrum slightly steeper than in the familiar Salpeter function; the stellar masses are in the ratio $1: 0.4: 0.16$, with the total mass in each group in the ratio $1: 2: 3$. This figure shows clearly how the heavier stars settle towards the center in a time of order $t_{r h}$. The rate at 
which stars accumulate in the halo or escape from the system per time interval equal to $t_{r h}$, is much the same as in the one-component case, except that this rate is about twice as high for the lighter stars as for the heavy ones. The stars of intermediate mass escape at about the same rate (within about 20\%) as the lightest stars, in general agreement with the results obtained for small- $N$ systems by Wielen (1968) and by Aarseth and Woolf (1972).

\subsection{CONTRACTION OF ISOTHERMAL CORE}

The initial contraction of the isothermal core for the three-component models is substantially faster than in the one-component system, as a result of the mass stratification. In all these models the contraction accelerates at the end, with the area of the spherical shells containing a fixed percentage of the total mass decreasing linearly with $t$ as before. The time, $t_{\text {coll }}$, from beginning to final collapse is shorter than before, with $t_{\text {coll }} / t_{r h}$ equal to about 3 , as compared with values between 12 and 19 for the one-component models.

For one-component models the mechanism producing contraction changed during the evolution of the system, with evaporation and halo buildup important initially and the gravothermal instability apparently dominant at the end. A similar transition apparently occurs for the three-component models. The rate of collapse at the end is about an order of magnitude too great to attribute to the energy transferred away from the heavies through equipartition, at a rate given by Equation (10). This result follows from the fact that the total fraction of the mass present in the form of light and intermediate stars together is very small in the central regions, - less than about $10 \%$ within the sphere containing $4 \%$ of the system's mass. However, the rate of collapse found in these models is consistent with that observed in the one-component models; the values of $1000 \eta$ for the three-component models range from 1.9 to 5.1, averaging 3.0, in rough accord with Equation (9). Furthermore, the density range within the inner half of the mass of the system again reaches some three orders of magnitude late in the collapse phase.

Important evidence on this question of collapse mechanism is obtained by a computer run in which encounters of heavy stars with each other were suspended arbitrarily at a later stage in the collapse. One would expect that this modification in the program would have no great effect on the tendency towards energy loss to the lighter stars, but should slow down the outwards energy flux important in the gravothermal instability. When this modification was made in one of the models, at a relatively advanced stage of evolution, the collapse of the very inner region (containing $2 \%$ of the mass) slowed down to about half its previous rate. Further out, the radius containing $10 \%$ of the mass stopped contracting entirely. While these results have not been explained in detail, they indicate that the transfer of energy from the heavy stars to the light stars is not the predominant mechanism responsible for the final collapse. In all probability, whatever detailed process accounts for the final collapse of onecomponent systems also plays an important part in the similar collapse of threecomponent clusters. 


\section{Results for Systems in Perturbing External Fields}

While the computations for isolated systems are of great physical interest, the attempt to explain actual clusters in our own Galaxy must take into account the external gravitational field to which these clusters are subject. The smoothed gravitational potential of the Galaxy itself is the largest such field, and the primary one which we shall consider. This field produces two effects. First, the cluster will undergo a quasi-steady tidal distortion, with stars escaping entirely whenever they enter certain 'escape regions'. Second, the cluster will gain internal energy as a result of changes in the local galactic field resulting from cluster motion. Neither of these effects is consistent with a spherically symmetric model of a cluster, but the resultant modifications of evolutionary development can be computed approximately with such symmetric models.

If we consider first the quasi-steady effects of the field, the tidal distortion can obviously not be taken into account when spherical symmetry is assumed. However, the effect of the tidal field on the rate of escape can be computed if we assume that any star reaching the distance $r_{e}$ from the cluster center will escape; the escape radius, $r_{e}$, can then be set equal to the minimum distance from the cluster center to the actual escape regions. In fact, stars in some directions can be stably bound to the cluster for substantial times at distances much exceeding $r_{e}$, but should escape in time when encounters deflect them into the true escape regions.

The most serious approximation associated with the assumption of spherical symmetry is that the angular momentum of each star remains constant in the absence of gravitational encounters between stars. The tidal field would be expected to make the velocities of the halo star more nearly isotropic. Hence a star diffusing towards zero energy, as a result of encounters during periodic passages through the isothermal core, might penetrate progressively less deeply into the core in its successive orbits; as pointed out by Aarseth and Woolf (1972), this effect would be expected to decrease the escape rate somewhat, offsetting at least partially the effect of reduced $r_{e}$. On the other hand, numerical orbit computations by Prata (1971) showed that an external tidal field did not in fact produce an isotropic distribution of velocities for $r$ less than about $\frac{1}{2} r_{e}$; possibly this result is produced by some third integral which constrains the orbit. The only firm conclusion indicated at present is that one must question the validity of results obtained with spherically symmetric models of tidally perturbed clusters.

We consider next effects produced by changes in the external field experienced by the cluster. As pointed out by Ostriker et al. (1972) rapid motion of a globular cluster through the galactic disc produces a transient compressive force perpendicular to the galactic plane, which can effectively heat a cluster; this effect is called a 'compressive shock'. This increase of internal energy can be computed for a spherically symmetric model, neglecting the transitory nonspherical distortions. The problem of the constancy of angular momentum in spherically symmetric models is unimportant in this case, since shock heating destroys this constancy in any case, and the computed models should be reasonably realistic. 
Monte Carlo models of systems with tidal cut-offs and with shock heating have been computed both for one-component (Spitzer and Chevalier, 1972 - Paper V) and three-component (Spitzer and Shull, 1975 - Paper VII) systems. Because of the many parameters involved, a full analysis of these results would be outside the scope of the present paper, and only a brief general review will be given.

As would be expected, the assumption of a finite escape radius, $r_{e}$, increases the escape rate, and also accelerates the initial contraction of one-component models, decreasing the time until the core collapses. If shock heating is assumed, the rate of escape is increased even further. This effect becomes particularly marked when a distribution of masses is present, since mass stratification forces the lighter stars out to larger radii, where shock heating is most effective. Thus imposing an escape radius as small as about $3 r_{h}$ increases the escape rate by about an order of magnitude above that for an isolated system. If moderate shock heating is assumed for a system with three mass components, another order of magnitude increase in $\xi_{e}$ is produced towards the end of the evolutionary development, leading to escape of about three fourths of the lightest stars during the time required to produce collapse of the core.

Since the effectiveness of shock heating for a particular star varies as the square of the distance from the cluster center, reasonable amounts of shock heating in existing globular clusters generally have no direct effect on collapse of the inner isothermal core once this collapse has started. However, shock heating can sometimes hasten the rate of collapse by affecting the initial rate of contraction. Thus a small amount of shock heating, by getting rid of stars in the outer part of a one-component cluster, can accelerate the rate of diffusion of stars from the core up towards small total energy, increasing the initial rate of contraction and decreasing $t_{\text {coll }}$. In a multicomponent system, on the other hand, when shock heating increases the rate of escape of the lighter stars, the number of these stars in the central core is diminished, and the rate at which the heavier stars lose energy through equipartition is somewhat reduced; this effect tends to offset the other, and shock heating has no marked effect on $t_{\text {coll }}$ in multi-component systems. With strong shock heating, such as may have existed at an earlier stage when clusters were more extended than they are now, complete dissolution of the system can be produced. Evidently with so many physical processes occurring a wide range of evolutionary histories becomes possible.

\section{Evolution of Globular Clusters}

To compare the theoretical models with observed clusters we must know the values of $t_{r h}$, the reference relaxation time (see Equation (5)). These values depend on the cluster mass, which has been determined from velocity dispersions measured in four globular clusters (Illingworth and Freeman, 1974, and references cited therein). If we adopt the value of $1.0 M_{\odot} / L_{\odot}$ for $M / L$ and use the observational data on clusters obtained by Peterson and King (1975) together with the analysis of these data by Ostriker et al. (1972), values of $t_{r h}$ can be computed for 32 clusters. The distribution of these values is shown in Figure 7. While these relaxation times cover a large spread, 
only one value exceeds $10^{10} \mathrm{yr}$. It seems likely that most clusters have existed for times substantially exceeding $t_{r n}$.

Two consequences seem to follow directly. Firstly, pronounced stratification of mass must have developed during the life of most of the clusters, provided that a wide initial distribution of masses was present initially. Secondly, if we continue to

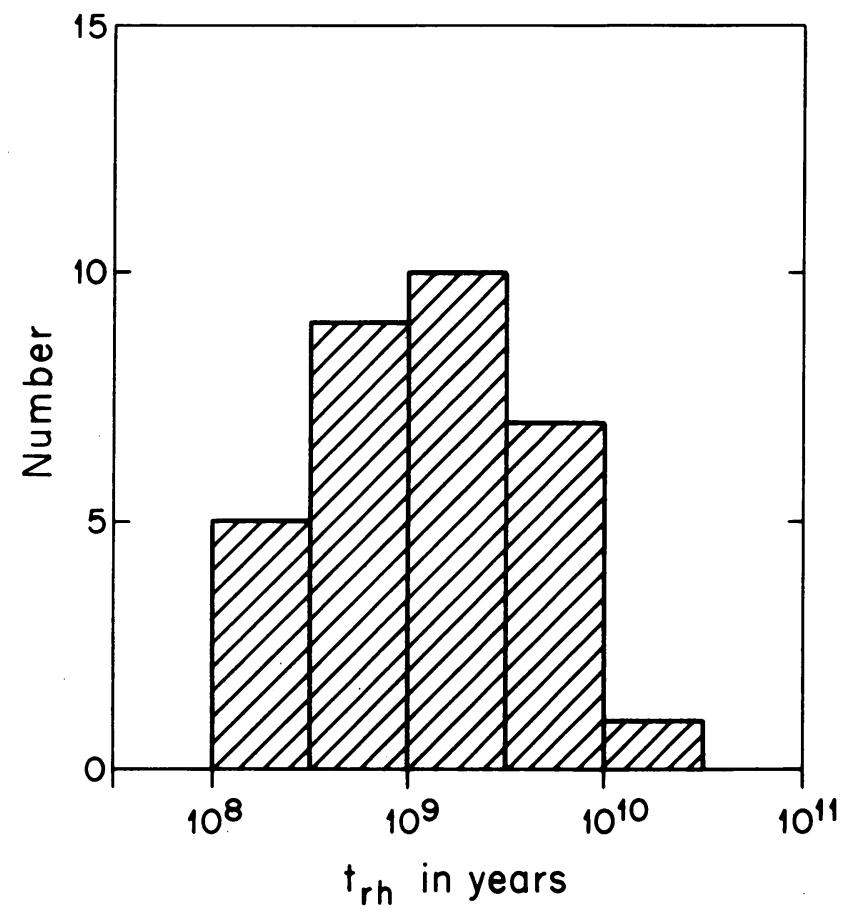

Fig. 7. Histogram showing the distribution of values of $t_{r h}$ in 32 clusters. The quantity $t_{r h}$ is the relaxation time for a star whose velocity equals the rms value for the cluster, and which is moving through a region of particle density equal to the mean value for the inner half of the cluster's mass.

ignore possible effects produced by an initial distribution of binaries, we must conclude that the cores of most clusters have gone through the collapse phase resulting from the gravothermal instability. We discuss each of these processes in turn.

Any stars of relatively low mass in a cluster should be relatively concentrated in the outer regions of each cluster. The prominent stars, all those above the red-giant turn-off point, all have presumably about the same mass, except perhaps for a short period before their death when mass.loss is significant. The mass of these stars in the upper part of the HR diagram is estimated by Ostriker et al. (1972) as $0.8 M_{\odot}$. Stars less massive than about $0.3 M_{\odot}$ will be fainter than the 26th magnitude even in the closest clusters $(m-M \geqslant 14)$, and hence too faint to detect. Whether these faint stars have escaped entirely, as suggested by Ostriker et al. (1972), or whether they are present in an extended halo around each cluster cannot be determined from the present evidence. Nearly complete escape of the lighter stars might have been possible 
at an earlier epoch, when the cluster was considerably more diffuse and the rate of shock heating was relatively more rapid. On the other hand, in the three-component Monte Carlo computations, the distribution of the heavier stars seems not unlike the observed clusters. If the presence of the lighter stars in these models is ignored and the virial theorem is applied to the heavier stars only, the derived mass exceeds only slightly the true total mass in the heavy stars; i.e., the self-attraction of the heavy stars is the dominant influence in accounting for their velocity dispersion. If a relatively massive halo of light stars is actually present in a globular cluster, the variation of velocity dispersion of the heavy stars with distance from the center should clearly be influenced, but observational evidence on so refined a point is entirely lacking. In any case it is clear that mass stratification, plus enhanced escape of the lighter stars, would seem to offer a reasonable interpretation for the observed clusters and their low apparent $M / L$ ratio.

Since collapse of an inner core appears to be an entirely possible event in the life of a cluster, one may inquire what the consequences of such a collapse might be. There seem to be two general possibilities for the final development of a collapsing core. On the one hand, the collapse may be terminated by the presence of binaries, which sink to lower and lower energies, ejecting other stars from the core if not from the cluster. As suggested by Heggie (1974) such binaries may form naturally as fewer and fewer stars become involved in the collapsing region. In this connection it is conceivable that a few tightly bound binaries present in the cluster may sink to the center of the system because of the higher combined mass of each binary; such binaries would then interact increasingly with the single stars composing the bulk of the contracting core.

On the other hand, if binaries are not present it would appear to be impossible to stop the collapse until the finite sizes of the stars become important. Physical collisions between stars will then occur. Since the stellar velocities at this time will be small compared to escape velocities from a stellar surface, coalescence between two colliding stars (Colgate, 1967; Sanders, 1970) will be more likely than disruption. Thus a few massive stars will form, and may either explode as supernovae or form binary systems which will tend to give up energy to single stars. If only a few supernovae were produced in each core collapse, the chance of observing such an event either in our own Galaxy or in neighboring systems would be very small, since supernovae formed in this way would constitute an extremely small addition to the overall supernova rate per galaxy.

In either case some of the mass of the core is likely to be ejected either as gas or as stars, and it is possible that the entire cluster may expand somewhat. It is difficult to see, however, how another contraction, leading to yet another collapse, can be avoided. If successive collapses follow each other and collisions between stars form an essential part of each cycle, these collapses may be expected to change their character as the stars gradually die and the heaviest stars are degenerate dwarfs. Formation of a massive black hole could be the final stage in cluster evolution. Clearly there are many challenging problems still to be explored in following the 
evolutionary development of a cluster to its ultimate, perhaps even apocalyptic, conclusion!

Parts of this summary were prepared and discussed at the 1974 Theoretical Astrophysics Workshop at the Aspen Center for Physics.

\section{References}

Aarseth, S. J.: 1968, Bull. Astron., Ser. 3 3, 105.

Aarseth, S. J.: 1975, 'Dynamical Evolution of Simulated Star Clusters. I: Isolated Systems' (preprint). Aarseth, S. J. and Woolf, N. J.: 1972, Astrophys. Letters 12, 159.

Albada, T. S. van: 1968, Bull. Astron. Inst. Neth. 19, 479.

Ambartsumian, V. A.: 1938, Ann. Leningrad State Univ. No. 22 (Astron. Series, Issue 4).

Antonov, V. A.: 1962, Bull. Leningrad State Univ. (Ser. Math. Mech. Astr.), No. 7, 135.

Chandrasekhar, S.: 1942, Principles of Stellar Dynamics, University of Chicago Press, Chicago, Ch. II, V. Colgate, S. A.: 1967, Astrophys. J. 150, 163.

Heggie, D. C.: 1974, 'The Importance of Binaries in Cluster Dynamics' (preprint).

Hénon, M.: 1967, Bull. Astron., Ser. 3 2, 91.

Hẻnon, M.: 1969, Astron. Astrophys. 2, 151.

Hénon, M.: 1971, Astrophys. Space Sci. 13, 284; 14, 151.

Hénon, M.: 1973, in L. Martinet and M. Mayor (eds.), Dynamical Structure and Evolution of Stellar Systems, Swiss Society of Astronomy and Astrophysics Third Advanced Course, Geneva Observatory, p. 183.

Hoerner, S. von: 1960, Z. Astrophys. 50, 184.

Hoerner, S. von: 1963, Z. Astrophys. 57, 47.

Illingworth, G. and Freeman, K. C.: 1974, Astrophys. J. Letters 188, L83.

Kamp, P. van de: 1971, Ann. Rev. Astron. Astrophys. 9, 103.

King, I. R.: 1958a, Astron. J. 63, 109.

King, I. R.: 1958b, Astron. J. 63, 114.

King, I. R.: 1965, Astron. J. 70, 376.

King, I. R.: 1966, Astron. J. 71, 64.

Larson, R. B.: 1970, Monthly Notices Roy. Astron. Soc. 150, 93.

Lynden-Bell, D. and Wood, R.: 1968, Monthly Notices Roy. Astron. Soc. 138, 495.

Michie, R. W.: 1963, Monthly Notices Roy. Astron. Soc. 125, 127.

Miller, R. H.: 1973, Astrophys. J. 180, 759.

Ostriker, J. P., Spitzer, L., and Chevalier, R. A.: 1972, Astrophys. J. Letters 176, L51.

Peterson, C. J. and King, I. R.: 1975, 'The Structure of Star Clusters. VI: Observed Radii and Structural Parameters in Globular Clusters' (preprint).

Prata, S.: 1971, Astron. J. 76, 1017.

Rosenbluth, M. N., MacDonald, W. M., and Judd, D. L.: 1957, Phys. Rev. 107, 351.

Sanders, R. H.: 1970, Astrophys. J. 162, 791.

Spitzer, L.: 1940, Monthly Notices Roy. Astron. Soc. 100, 396.

Spitzer, L.: 1969, Astrophys. J. Letters 158, L139.

Spitzer, L. and Harm, R.: 1958, Astrophys. J. 127, 544.

Spitzer, L. and Hart, M. H.: 1971a, Astrophys. J. 164, 399 (Paper I).

Spitzer, L. and Hart, M. H.: 1971b, Astrophys. J. 166, 483 (Paper II).

Spitzer, L. and Shapiro, S. L.: 1972, Astrophys. J. 173, 529 (Paper III).

Spitzer, L. and Thuan, T. X.: 1972, Astrophys. J. 175, 31 (Paper IV).

Spitzer, L. and Chevalier, R. A.: 1973, Astrophys. J. 183, 565 (Paper V).

Spitzer, L. and Shull, J. M.: 1975, 'Random Gravitational Encounters and the Evolution of Spherical Systems. VII : Systems with Several Mass Groups' (preprint).

Wielen, R.: 1968, Bull. Astron., Ser. 3 3, 127.

\section{DISCUSSION}

Lecar: What is the advantage of integrating the stellar orbits (in contrast to Hénon's method)? 
Spitzer: The main advantage is that you can determine the escape rate. To evaluate this rate it is necessary to avoid going to the limit of large $N$ at the beginning. When $N$ is infinite, escape of stars becomes indistinguishable from accumulation in the far halo. For some purposes you don't really care which is which, in which case this large- $N$ limit is appropriate to start with. But if you are interested in finding the actual escape rate, then you must keep $N$ finite until you determine this rate, and then let $N$ increase. As it turns out, the escape rate determined in this way is virtually independent of $N$.

Ipser: What is the mass to radius ratio of your core? How does it vary as the core contracts?

Spitzer: The ratio of mass to radius does not change very rapidly. This ratio varies with the mean square velocity, which goes up but rather slowly. So the ratio increases slightly with time.

King: In your first comparison of envelope profile with the $r^{-3.5}$ law it seemed to me that the slope was more like $r^{-3}$, which is more like what is observed. In particular, Hubble's law for elliptical galaxies, which has this slope, has always been an enigma.

Spitzer: For model F, you start with no halo at all, so it is quite difficult to build up a halo; as a result the envelope is rather curved on a $\log -\log$ plot of $\varrho$ against $r$. In Model $\mathrm{D}$ a conspicuous halo is produced in the initial collapse, and a well developed halo exists throughout the evolutionary life of the system; for this halo the $r^{-3.5}$ relationship fitted the density quite well. We tried to fit an inverse cube law to the density, but were unable to do so.

King: Can you say something about the economics of your calculations? How much does it cost to run a model, and how many man years have gone into the programming?

Spitzer: One typical evolutionary model on the Princeton IBM 360/91 costs us about \$600. The computing program was written and applied by one graduate student during about half a year; in successive years three other graduate students have extended the program, again each devoting about half a year to this research effort. 